\title{
Isolated Superior Ophthalmic Vein Thrombosis Associated with Orbital Cellulitis: Case Report
}

\author{
(D) Zeynep Ozer Ozcan, (1) Alper Mete \\ Department of Ophtalmology, Gaziantep University, Gaziantep, Turkey
}

\begin{abstract}
Superior ophthalmic vein thrombosis (SOVT) is a rare clinical entity that may be associated with sino-orbital disease. The clinical presentation of SOVT may include signs of venous congestion, such as unilateral ptosis, chemosis, ophthalmoplegia, and eyelid swelling, with or without fundus findings. This case report describes a case of SOVT associated with orbital cellulitis diagnosed with magnetic resonance imaging and treated using anticoagulant therapy, antibiotherapy, and a corticosteroid. In the presence of orbital cellulitis, clinicians should always keep the possibility of SOVT in mind, as it may result in mortality and visual loss if not diagnosed early and given appropriate treatment without delay.
\end{abstract}

Keywords: Anticoagulant therapy, orbital cellulitis, superior ophthalmic vein thrombosis.

\section{Introduction}

Superior ophthalmic vein thrombosis (SOVT) is a rare clinical condition that may occur as a result of sino-orbital disease, trauma, neoplasm, or hypercoagulation (I). The clinical presentation of SOVT may include signs of venous congestion, such as unilateral ptosis, chemosis, ophthalmoplegia, and eyelid swelling, with or without fundus findings (2). These symptoms also appear in cases of orbital cellulitis and cavernous sinus thrombosis (CST). Additionally, these 3 entities can occur together, which can make a clinical differential diagnosis difficult (3). SOVT is considered an early symptom of CST, which can result in pituitary insufficiency, hemiparesis, and death, so early diagnosis and treatment are critical (4). This is a case report of SOVT, which has a potential risk of mortality and visual loss, associated with orbital cellulitis.

\section{Case Report}

A 52-year-old female patient presented at our clinic with a history of thyroidectomy related to Graves' disease, diabetes, and hypertension. The symptoms were headache; purulent nasal discharge; and pain, swelling, and motility restriction in the right eye that had been ongoing for I week. The visual acuity finding was $20 / 20$ in both eyes with a lack of a relative afferent pupillary defect. Her Ishihara test results of color vision were normal and no visual field defect was determined in a confrontation test. The intraocular pressure measurement was $36 \mathrm{mmHg}$ in the right eye and $18 \mathrm{mmHg}$ in the left eye. Hertel's exophthalmometry revealed proptosis of $23 \mathrm{~mm}$ and $17 \mathrm{~mm}$ in the right and left eye, respectively. Slit-lamp examination showed eyelid edema, conjunctival injection, chemosis, episcleral vascular engorgement, and tortuosity (Fig. IA, B). A fundus exami- 

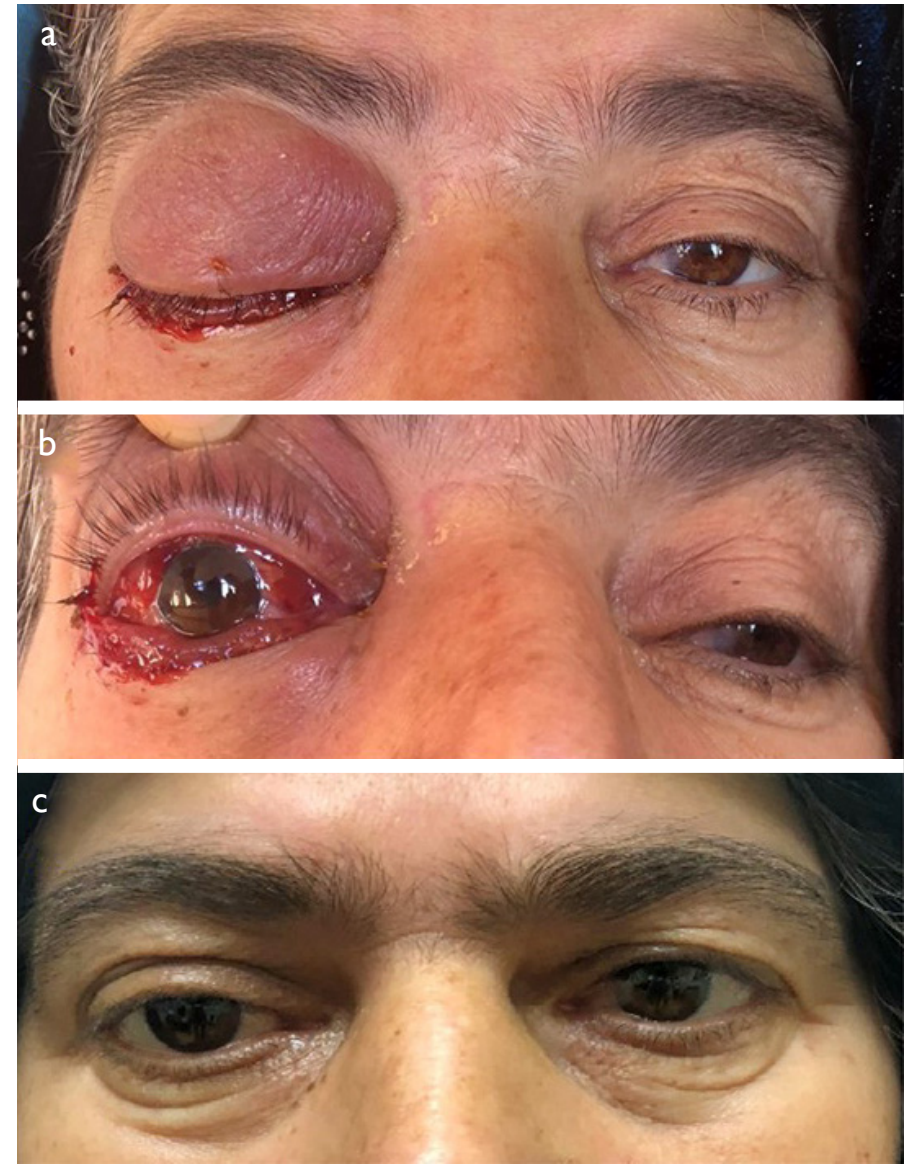

Figure I. (a) and (b) Images of the patient with unilateral ptosis, eyelid edema, conjunctival injection, chemosis, episcleral vascular engorgement, and tortuosity; (c) an image taken of the same patient on the $10^{\text {th }}$ day of treatment.

nation was bilaterally normal. Extraocular motility was limited in all directions.

A paranasal sinus computed tomography (CT) examination was performed to clarify the etiology, and right proptosis, periorbital soft tissue inflammation, ipsilateral ethmoidal sinusitis, and dilatation in the right superior ophthalmic vein (SOV) were diagnosed (Fig. 2). Magnetic resonance imaging (MRI) indicated a thickened and thrombosed SOV lumen, but a normal right cavernous sinus (Fig. 3).

Blood culture and conjunctival swab samples were negative. The thyroid-stimulating hormone level value was .37 $\mathrm{mU} / \mathrm{L}$ (laboratory range: $0.34-5.6 \mathrm{mU} / \mathrm{L}$ ) and cardiovascular disease panel results were normal. Levothyroxine $(25 \mathrm{mg})$, topical dorzolamide-timolol $0.5 \%$, latanoprost, a wide-spectrum antibiotic combination (systemic meropenem and linezolid) were administered, as well as anticoagulant therapy (ACT) (low-molecular-weight heparin $2 \times 6000 \mathrm{lU}$ was initiated after heparin infusion monitoring with an activated partial thromboplastin time test), and oral methylprednisolone (64 $\mathrm{mg})$. After 10 days of treatment, the eyelid edema, conjunctival injection, chemosis, episcleral vascular engorgement, and

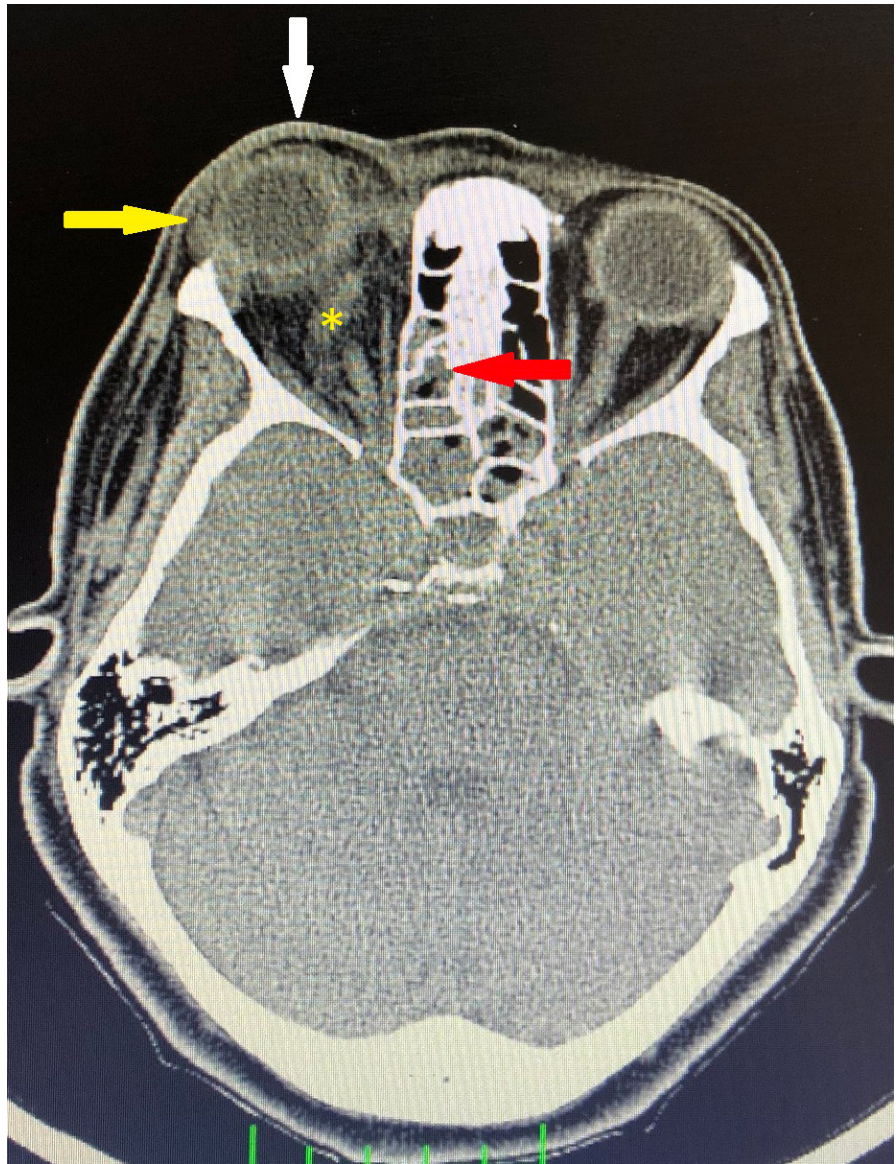

Figure 2. An axial computed tomography scan illustrating proptosis (white arrow), soft tissue inflammation (yellow arrow), dilatation in the right superior ophthalmic vein (asterisk), and ethmoidal sinusitis (red arrow).

tortuosity were reduced, and eye movements were free in all directions (Fig. IC).

\section{Discussion}

SOV valves primarily provide venous drainage of the eye. There may be septic or aseptic causes that result in SOVT. Aseptic causes can include stasis of blood flow associated with anatomical or systemic causes, traumatic vessel wall damage, and hypercoagulability disorders (5). Septic causes can include paranasal sinus infection (most common), in addition to orbital, dental, and facial infections (6). SOVT is an uncommon disease that can lead to vision loss associated with compartment syndrome or secondary glaucoma $(7,8)$. The SOV is thought to be the first means of ethmoidal sinusitis becoming cavernous sinus (9). The most feared complication of SOVT is cavernous sinus, due to the high risk of mortality.

Contrast-enhanced CT or MRI can be used for diagnosis. Ambiguity at the outer border of the vessel due to perivascular edema, thickening of the lumen, or a filling defect can be resolved with contrast-enhanced CT. Similar to contrast- 

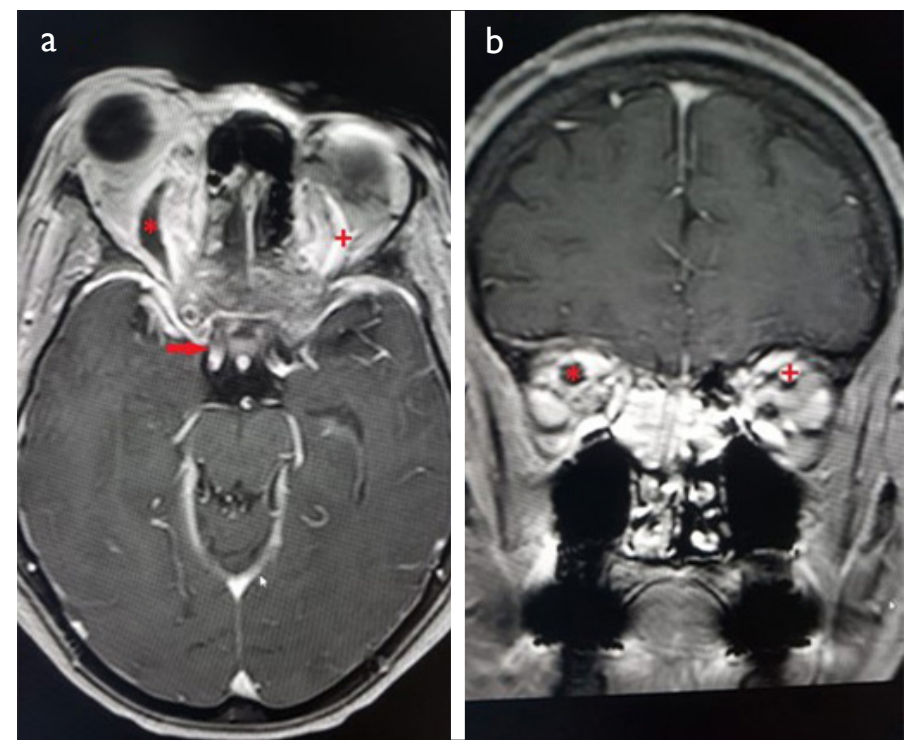

Figure 3. (a) and (b) Axial and coronal contrast-enhanced magnetic resonance imaging sections revealing a dilated right superior ophthalmic vein with a filling defect (asterisk) and a normal left superior ophthalmic vein (plus sign). Red arrow indicates normal right cavernous sinus.

enhanced CT, MRI may show filling defects in the SOV lumen (Fig. 3). Other signs include proptosis, expansion of intraorbital adipose tissue, and extraocular muscle edema due to venous congestion. Unlike MRI, CT may not show SOVT and/ or cavernous sinus thrombosis in the early period. Therefore, many authors consider MRI to be more useful in the diagnosis (I0). In the present case, we performed a paranasal sinus CT to elucidate the etiology with a preliminary diagnosis of orbital cellulitis. We found ethmoidal sinusitis and dilatation in the right SOV. A contrast-enhanced MRI was then performed, which confirmed SOVT and excluded CST.

Unfortunately, the literature on the treatment of SOVT consists only of case reports. Once the clinician clarifies the etiology, targeted treatment can be performed.

Treatment may include antibiotics, steroids, ACT, and/or surgery. Antibiotic therapy should be initiated according to the possible source of infection while waiting for the results of an antibiogram. Anaerobic or aerobic microorganisms, such as Staphylococcus aureus, are generally detected in the case of orbital cellulitis associated with ethmoidal sinusitis (II). There is no consensus on the duration of antibiotic therapy. In our case, due to the negative blood culture and conjunctival swab sample findings, we administered a widespectrum antibiotic for 10 days. The evidence regarding steroid use remains insufficient in cases of SOVT.

There is no consensus on the use of ACT, due to the risk of hemorrhage, but recent literature reports show that the risk of hemorrhage is low in many patients. Although there is a lack of homogeneity regarding either treatment modalities or patient profiles in the literature, Weerashing- he et al.(I2) found that mortality was decreased in patients with CST who underwent ACT. Although the use of ACT is recommended, currently the dose and duration of treatment are not optimized. In general, ACT is considered after a risk-benefit analysis. The risk of hemorrhage (particularly retroperitoneal hemorrhage) should not be forgotten during the use of ACT and the patient should be kept under close follow-up (13). Due to the absence of any susceptibility to bleeding in our case, we started anticoagulant therapy early. Surgical treatment may be considered in cases of sinusitis, orbital abscess, or dental infection.

In conclusion, SOVT is a rare disease, but one which may result in vision loss or life-threatening complications if orbital cellulitis degenerates into a cavernous sinus. Clinicians should always keep this rare complication in mind and provide the appropriate treatment without delay.

\section{Disclosures}

Informed consent: Written informed consent was obtained from the patient for the publication of the case report and the accompanying images.

Peer-review: Externally peer-reviewed.

Conflict of Interest: None declared.

Authorship Contributions: Involved in design and conduct of the study (ZOO, AM); preparation and review of the study (ZOO, AM); data collection (ZOO).

\section{References}

I. Sorrentino D, Taubenslag KJ, Bodily LM, Duncan K, Stefko T, Yu JY. Superior ophthalmic vein thrombosis: A rare complication of Graves' orbitopathy. Orbit 2018;37:175-8. [CrossRef]

2. Walker JC, Sandhu A, Pietris G. Septic superior ophthalmic vein thrombosis. Clin Exp Ophthalmol 2002;30:144-6. [CrossRef]

3. van der Poel NA, de Witt KD, van den Berg R, de Win MM, Mourits MP. Impact of superior ophthalmic vein thrombosis: a case series and literature review. Orbit 2019;38:226-32. [CrossRef]

4. Cumurcu T, Demirel S, Keser S, Bulut T, Cavdar M, Doğan M, Saraç K. Superior ophthalmic vein thrombosis developed after orbital cellulitis. Semin Ophthalmol 2013;28:58-60. [CrossRef]

5. Levin MH, Moss HE, Pineles SL, Bagley LJ, Heuer GG, Zager $\mathrm{EL}$, et al. Orbital congestion complicating treatment of cerebral vascular anomalies. World Neurosurg 2014;82:239.e I3-7.

6. Mandić JJ, Mandić K, Mrazovac D. Superior Ophthalmic Vein Thrombosis with Complete Loss of Vision as a Complication of Autoimmune and Infective Conditions. Ocul Immunol Inflamm 2018;26:1066-8. [CrossRef]

7. Lim LH, Scawn RL, Whipple KM, Oh SR, Lucarelli MJ, Korn BS, et al. Spontaneous superior ophthalmic vein thrombosis: a rare entity with potentially devastating consequences. Eye (Lond) 20I4:28:348-5I. [CrossRef]

8. Ladner TR, Davis BJ, He L, Mawn LA, Mocco J. Transorbital superior ophthalmic vein sacrifice to preserve vision in ocular 
hypertension from aseptic cavernous sinus thrombosis. J Neurointerv Surg 2015;7:e39. [CrossRef]

9. Berenholz L, Kessler A, Shlomkovitz N, Sarfati S, Segal S. Superior ophthalmic vein thrombosis: complication of ethmoidal rhinosinusitis. Arch Otolaryngol Head Neck Surg 1998; 124:95-7.

10. Velasco e Cruz AA, Demarco RC, Valera FC, dos Santos AC, Anselmo-Lima WT, Marquezini RM. Orbital complications of acute rhinosinusitis: a new classification. Braz J Otorhinolaryngol 2007;73:684-8. [CrossRef]
II. Osborn MK, Steinberg JP. Subdural empyema and other suppurative complications of paranasal sinusitis. Lancet Infect Dis 2007;7:62-7. [CrossRef]

12. Weerasinghe D, Lueck CJ. Septic Cavernous Sinus Thrombosis: Case Report and Review of the Literature. Neuroophthalmology 2016;40:263-76. [CrossRef]

13. Bhatia K, Jones NS. Septic cavernous sinus thrombosis secondary to sinusitis: are anticoagulants indicated? A review of the literature. J Laryngol Otol 2002; I 16:667-76. [CrossRef] 